\title{
Adsorption-controlled growth and properties of epitaxial SnO films
}

\author{
Antonio B. Mei, ${ }^{1,2,{ }^{*}}$ Ludi Miao, ${ }^{3}$ Matthew J. Wahila, ${ }^{4}$ Guru Khalsa, ${ }^{1}$ Zhe Wang, ${ }^{5}$ Matthew Barone, ${ }^{1}$ Nathaniel J. Schreiber, ${ }^{1}$ \\ Lindsey E. Noskin, ${ }^{1}$ Hanjong Paik, ${ }^{1,6}$ Thomas E. Tiwald, ${ }^{7}$ Qiye Zheng, ${ }^{2,8,9}$ Richard T. Haasch, ${ }^{2}$ Davide G. Sangiovanni, ${ }^{10,11}$ \\ Louis F. J. Piper, ${ }^{4}$ and Darrell G. Schlom ${ }^{1,12}$ \\ ${ }^{1}$ Department of Materials Science and Engineering, Cornell University, Ithaca, New York 14853, USA \\ ${ }^{2}$ Department of Materials Science and the Materials Research Laboratory University of Illinois, \\ 104 South Goodwin, Urbana, Illinois 61801, USA \\ ${ }^{3}$ Laboratory of Atomic and Solid State Physics, Cornell University, Ithaca, New York 14853, USA \\ ${ }^{4}$ Department of Physics, Applied Physics and Astronomy, Binghamton University, Binghamton, New York 13902, USA \\ ${ }^{5}$ School of Applied and Engineering Physics, Cornell University, Ithaca, New York 14853, USA \\ ${ }^{6}$ Platform for the Accelerated Realization, Analysis, and Discovery of Interface Materials (PARADIM), \\ Cornell University, Ithaca, New York 14853, USA \\ ${ }^{7}$ J.A. Woollam Co., Lincoln, Nebraska 68508, USA \\ ${ }^{8}$ Lawrence Berkeley National Laboratory, Berkeley, California 94720-1740, USA \\ ${ }^{9}$ Mechanical Engineering, University of California at Berkeley, California 94720-1740, USA \\ ${ }^{10}$ Department of Physics, Chemistry and Biology (IFM), Linköping University, SE-581 83 Linköping, Sweden \\ ${ }^{11}$ ICAMS, Ruhr-Universität Bochum, D-44780 Bochum, Germany \\ ${ }^{12}$ Kavli Institute at Cornell for Nanoscale Science, Ithaca, New York 14853, USA
}

(Received 28 May 2019; published 21 October 2019)

\begin{abstract}
When it comes to providing the unusual combination of optical transparency, $p$-type conductivity, and relatively high mobility, $\mathrm{Sn}^{2+}$-based oxides are promising candidates. Epitaxial films of the simplest $\mathrm{Sn}^{2+}$ oxide, $\mathrm{SnO}$, are grown in an adsorption-controlled regime at $380{ }^{\circ} \mathrm{C}$ on $\mathrm{Al}_{2} \mathrm{O}_{3}$ substrates by molecular-beam epitaxy, where the excess volatile $\mathrm{SnO}_{x}$ desorbs from the film surface. A commensurately strained monolayer and an accompanying van der Waals gap is observed near the substrate interface, promoting layers with high structural perfection notwithstanding a large epitaxial lattice mismatch $(-12 \%)$. The unintentionally doped films exhibit $p$-type conductivity with carrier concentration $2.5 \times 10^{16} \mathrm{~cm}^{-3}$ and mobility $2.4 \mathrm{~cm}^{2} \mathrm{~V}^{-1} \mathrm{~s}^{-1}$ at room temperature. Additional physical properties are measured and linked to the $\mathrm{Sn}^{2+}$ valence state and corresponding lone-pair charge-density distribution.
\end{abstract}

DOI: 10.1103/PhysRevMaterials.3.105202

\section{INTRODUCTION}

The amalgamation of high electrical conductivity and optical transparency within transparent conducting oxides (TCOs) is critically important for the development of next generation, high efficiency photovoltaics and interactive transparent electronics [1]. To date, designing TCOs has mainly involved doping wide-band-gap semiconductors. While this methodology has successfully produced $n$-type materials with low electron effective masses and high mobilities [2-4], the synthesis of $p$-type TCOs with equally desirable properties has been considerably more challenging.

Recent high-throughput searches founded on first-principle calculations identify $\mathrm{Sn}^{2+}$-based oxides as promising candidates for optically transparent $p$-type conductors [5]. In addition to exhibiting low hole effective masses and large band gaps, these compounds also display attractive dopant characteristics in which shallow acceptor states give rise to intrinsic $p$-type behavior that remain uncompensated by the energetically costly formation of oxygen-vacancy donor states [5-7]. Unfortunately, tin robustly prefers the $\mathrm{Sn}^{4+}$ oxidation state, making the stabilization of $\mathrm{Sn}^{2+}$-based oxides difficult [8-10].

*amei2@illinois.edu
Of all compounds involving $\mathrm{Sn}^{2+}$, stannous oxide $(\mathrm{SnO})$, with its simple binary structure, represents a quintessential model system to investigate and demonstrate valence stabilization in high-quality single-crystalline form. $\mathrm{SnO}$ is fundamentally important for its pressure-induced insulator-metal phase transition [11], which concomitantly kindles superconductivity [11,12] as observed in isostructural FeSe [13,14] and is technologically relevant for next-generation computing [15-18] and energy-sustainable applications [19,20]. Despite its simple structure and unique properties, the quality of $\mathrm{SnO}$ films reported in the literature varies greatly [15,21-27]. Part of the challenge with obtaining high-quality $\mathrm{SnO}$ is stabilizing $\mathrm{Sn}^{2+}$ over $\mathrm{Sn}^{4+}$ [28,29]. Indeed, thermodynamic phase diagrams omit $\mathrm{SnO}$ considering it metastable and to disproportionate to $\mathrm{Sn}$ and $\mathrm{SnO}_{2}$ [30].

Strategies to stabilize $\mathrm{Sn}^{2+}$ include using metal-organic precursors [28] and exploiting the higher vapor pressure of $\mathrm{SnO}$ suboxides over $\mathrm{SnO}_{2}$ [4,21-25,31]. Here, we adopt the latter approach and present a study of the growth and properties of $\mathrm{SnO}$ films produced using molecular-beam epitaxy. The single-crystalline layers are deposited in a spiral growth mode at temperatures compatible with back-end-of-line fabrication processes. After establishing that these epitaxial $\mathrm{SnO}$ films have the highest structural perfection as well as 

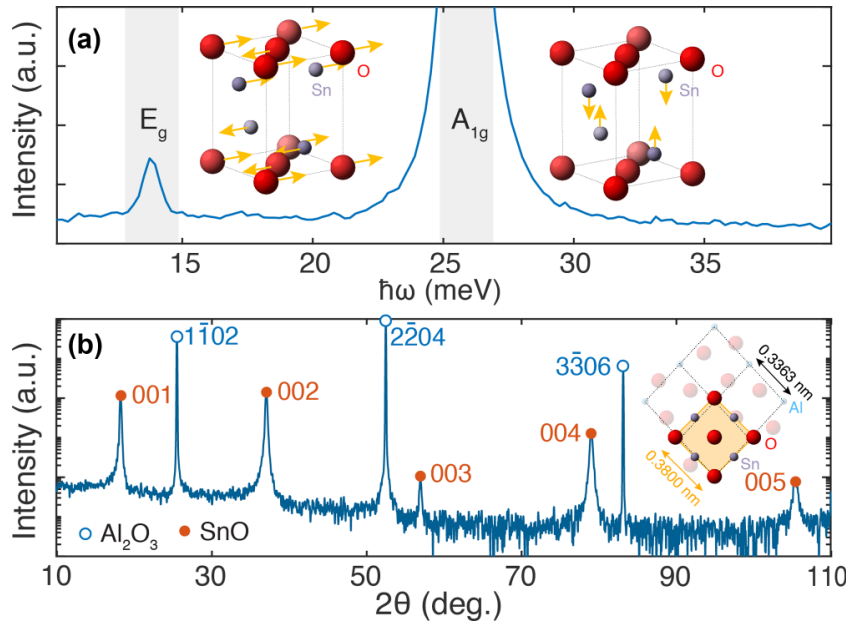

FIG. 1. Phase-pure litharge $\mathrm{SnO} / \mathrm{Al}_{2} \mathrm{O}_{3}(1 \overline{1} 02)$ films produced via molecular-beam epitaxy. (a) Backscattered Raman Stokes spectrum and (b) XRD $\theta-2 \theta$ scan establishing phase-pure litharge $\mathrm{SnO} / \mathrm{Al}_{2} \mathrm{O}_{3}(1 \overline{1} 02)$ films. The inset depict atomic displacement patterns corresponding to Raman-active vibrational modes (a) and the film/substrate orientational relationship (b).

the lowest background carrier concentrations reported to date, we proceed to investigate the electronic properties of $\mathrm{SnO}$ by combining spectroscopic measurements with first-principles calculation results.

\section{RESULTS AND DISCUSSION}

\section{A. Phase identification}

$\mathrm{SnO}$ films are grown on $r$-plane $\mathrm{Al}_{2} \mathrm{O}_{3}(1 \overline{1} 02)$ substrates using molecular-beam epitaxy in a Veeco GEN10 stainless-steel ultrahigh-vacuum system (base pressure $=$ $1 \times 10^{-8}$ Torr) under a background $\mathrm{O}_{2}$ partial pressure of $5 \times 10^{-7}$ Torr. $\mathrm{SnO}$ is supplied from an $\mathrm{SnO}_{2}$-containing (99.996\% purity, Alfa Aesar) effusion cell operating near $950^{\circ} \mathrm{C}$. In situ reflection high-energy electron diffraction patterns demonstrate that layers deposited at a substrate temperature $T_{s}$ below $370^{\circ} \mathrm{C}$ are amorphous and that no deposition occurs above $400^{\circ} \mathrm{C}$. At high homologous growth temperatures, adsorbed $\mathrm{SnO}_{x}$ species return to the gas phase due to their low sticking probabilities rather than accumulating on the growth surface [32,33]. Films grown between $370^{\circ} \mathrm{C} \leqslant T_{s} \leqslant 400^{\circ} \mathrm{C}$ are crystalline and represent the main focus of this article. The following discussion is for a $\mathrm{SnO}$ layer deposited at $380^{\circ} \mathrm{C}$ on $r$-plane $\mathrm{Al}_{2} \mathrm{O}_{3}(1 \overline{1} 02)$ in a background $\mathrm{O}_{2}$ partial pressure of $5 \times 10^{-7}$ Torr.

The crystallographic phase of $\mathrm{SnO} / \mathrm{Al}_{2} \mathrm{O}_{3}(1 \overline{1} 02)$ layers is established using Raman spectroscopy and $\mathrm{x}$-ray diffraction (XRD). Figure 1(a) is a representative backscattered Stokes spectrum $^{1}$ [34]. The peaks at $\hbar \omega=13.7$ and $25.8 \mathrm{meV}$ correspond to symmetry-allowed vibrational excitations unique

\footnotetext{
${ }^{1}$ Raman spectra are collected in a confocal microscope using a $100 \times$ objective $(\mathrm{NA}=0.90)$, a $2.54-\mathrm{eV}(488-\mathrm{nm})$ laser linearly polarized along $\mathrm{SnO}[100]$, and a parallel analyzer configuration, i.e., $\bar{z}(x x) z$.
}

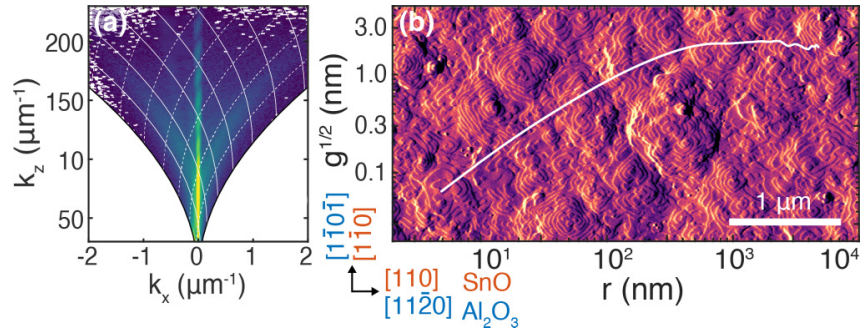

FIG. 2. Spiral growth of fully dense $\mathrm{SnO} / \mathrm{Al}_{2} \mathrm{O}_{3}(1 \overline{1} 02)$ films. (a) XDS map exhibiting diffuse wings and a decay in specular intensity that is indicative of fully dense films $\left(6.2 \mathrm{~g} / \mathrm{cm}^{3}\right)$ and atomically smooth surfaces (1.0 nm roughnesses). (b) AFM amplitude image showing shallow spiral growth mounds. The overlaid heightdifference correlation function has a presaturation slope which is consistent with high adatom diffusivity.

to a specific crystallographic phase. To identify the phase, we decompose zone-center phonon modes for different tin oxide phases into irreducible representations and compute $[35,36]$ the energy $\hbar \omega$ and differential scattering cross-section $d \sigma / d \Omega$ of each symmetric representation using density functional perturbation theory. For litharge $\mathrm{SnO}$, the analysis yields four Raman-active representations with energies spanning 14.2 $\left(E_{g}\right), 25.8\left(A_{1 g}\right), 42.6\left(B_{1 g}\right)$, and $56.3 \mathrm{meV}\left(E_{g}^{\prime}\right)$. Computed $d \sigma / d \Omega$ values indicate that the activity of the latter two modes, $B_{1 g}$ and $E_{g}^{\prime}$, are strongly suppressed, consistent with their absence in the recorded spectrum. The former two modes, for which corresponding atomic displacement patterns are illustrated in Fig. 1(a), exhibit energies that are in excellent agreement with observed peak positions. Collectively, the agreement between the theoretical and experimental findings indicate that our layers are $\mathrm{SnO}$ with the litharge crystallographic structure.

Figure 1(b) is an XRD $\theta-2 \theta$ scan acquired from the same $\mathrm{SnO} / \mathrm{Al}_{2} \mathrm{O}_{3}(1 \overline{1} 02)$ film using $\mathrm{Cu} K_{\alpha 1}$ radiation. Between $2 \theta=$ $10^{\circ}-110^{\circ}$, only one family of film reflections is observed. The peaks are indexed as $\mathrm{SnO} 00 l$, yielding [37] an out-ofplane lattice parameter $c=0.4840 \pm 0.0005 \mathrm{~nm}$, in agreement with $0.4841 \mathrm{~nm}$ refined [38] from powder samples [39]. The absence of other reflections corroborate Raman findings, establishing phase-pure $\mathrm{SnO}$ layers with the litharge crystal structure.

\section{B. Growth mechanism}

X-ray diffuse scattering (XDS) and atomic force microscopy (AFM) experiments are employed to determine the growth modality of litharge $\mathrm{SnO} / \mathrm{Al}_{2} \mathrm{O}_{3}(1 \overline{1} 02)$ layers. Diffuse scattering maps, including Fig. 2(a), exhibit specular intensity oscillations [40] along $k_{x}=0$ which decay slowly with increasing scattering vector $k_{y}$ as well as pronounced wings [41], which appear at a fixed tilt from the sample surface. Modeling [42] the intensity variation establishes that the film surface is atomically smooth with a roughness of $\rho_{\mathrm{rms}}=1.0 \mathrm{~nm}$ and that the $\mathrm{SnO}$ layer is fully dense with a mass density of $\rho_{\mathrm{d}}=6.2 \mathrm{~g} / \mathrm{cm}^{3}$. Fully dense films are consistent with smooth surfaces since shallow growth mounds result in minimal atomic shadowing during film deposition. 


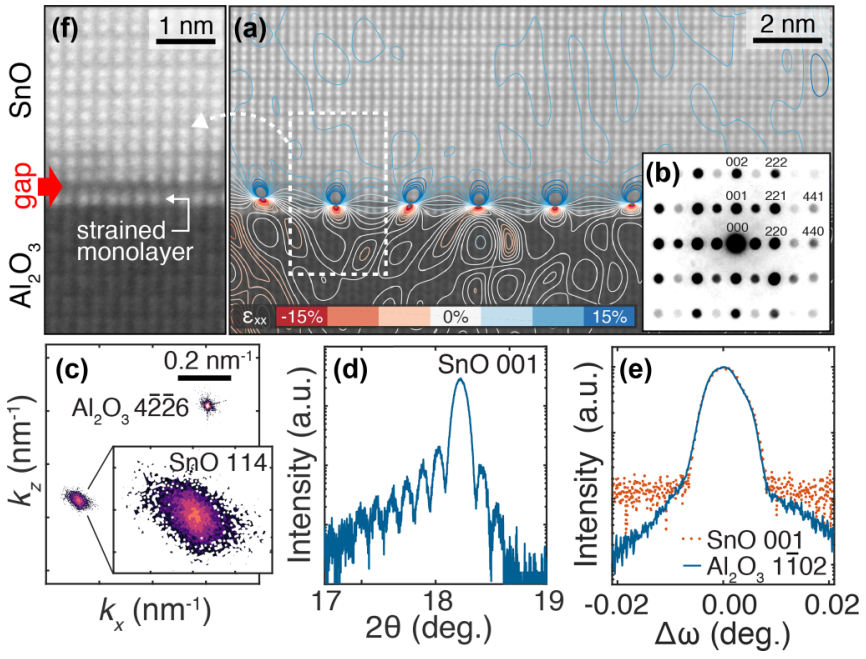

FIG. 3. Structural perfection of semicoherent $\mathrm{SnO} / \mathrm{Al}_{2} \mathrm{O}_{3}(1 \overline{1} 02)$ films. (a) STEM image acquired along the $\mathrm{Al}_{2} \mathrm{O}_{3}$ [1 $\left.1 \overline{1} 0 \overline{1}\right]$ zone axis near the $\mathrm{SnO} / \mathrm{Al}_{2} \mathrm{O}_{3}$ interface. Misfit dislocations are exposed by the overlaid in-plane strain isocontours. (b) NBD pattern of the film region. Indexed reflections indicate an $(001)_{\mathrm{SnO}} \|(1 \overline{1} 02)_{\mathrm{Al}_{2} \mathrm{O}_{3}}$ and $[110]_{\mathrm{SnO}} \|[11 \overline{2} 0]_{\mathrm{Al}_{2} \mathrm{O}_{3}}$ epitaxial relationship. (c) $\mathrm{RSM}$ of $\mathrm{SnO}$ 114 and $\mathrm{Al}_{2} \mathrm{O}_{3} 4 \overline{2} \overline{2} 6$ peaks evincing overlayer relaxation. (d) $\theta-2 \theta$ $\mathrm{XRD}$ scan in the vicinity of the $\mathrm{SnO} 001$ peak. (e) Superimposed XRD rocking curve scans of the $\mathrm{SnO} 001$ and $\mathrm{Al}_{2} \mathrm{O}_{3} 1 \overline{1} 02$ peaks, establishing substrate-limited film structural perfection. The full width at half maximum of both film and substrate $\omega$-rocking curve peaks is $0.007^{\circ}$ ( $25 \mathrm{arcsec}$ ). (f) Higher magnification STEM image highlighting a $0.40 \pm 0.03$-nm-wide gap that develops, separating a commensurately strained monolayer of the $\mathrm{SnO}$ film from the remainder of the fully relaxed $\mathrm{SnO}$ layer. The gap, which is a signature of van der Waals epitaxy, pins dislocations as misfits near the film/substrate interface, promoting the growth of films with high structural perfection.

AFM amplitude images, such as the one shown in Fig. 2(b), demonstrate that the film surface is composed of growth mounds with unit-cell-high terraces originating from adatom step-edge barriers [43,44]. The steps orient predominately along $\mathrm{SnO}\langle 100\rangle$ and occasionally terminate at screw dislocations (areal screw dislocation density $5 \times 10^{9} \mathrm{~cm}^{-2}$ ). Overlaid on Fig. 2(b) is $g^{1 / 2}(r)$ the surface height-difference correlation function, which statistically quantifies the surface roughness as a function of $r$ distance on the sample surface [45]. The analysis reveals extremely shallow mounds with aspect ratio of 0.001 and a surface morphology that is consistent with a high degree of adatom diffusion during film growth. Together, the XDS and AFM results indicate that the synthesis of $\mathrm{SnO}$ films on $\mathrm{Al}_{2} \mathrm{O}_{3}(1 \overline{1} 02)$ proceeds in a spiral growth mode.

\section{Film structure}

The nanostructure of $\mathrm{SnO} / \mathrm{Al}_{2} \mathrm{O}_{3}(1 \overline{1} 02)$ films are investigated using scanning transmission electron microscopy (STEM). A STEM micrograph acquired along the $\mathrm{Al}_{2} \mathrm{O}_{3}$ [11101] zone axis, near the film/substrate interface is presented in Fig. 3(a). The film region exhibits a pattern consistent with the litharge structure projected along the $\mathrm{SnO}[1 \overline{1} 0]$ zone axis. Indexing nanobeam diffraction (NBD) patterns collected from the film [Fig. 3(b)] confirms the overlayer orientation and, furthermore, establishes an $(001)_{\mathrm{SnO}} \|(1 \overline{1} 02)_{\mathrm{Al}_{2} \mathrm{O}_{3}}$ and $[110]_{\mathrm{SnO}} \|[11 \overline{2} 0]_{\mathrm{Al}_{2} \mathrm{O}_{3}}$ epitaxial relationship. Together with XRD pole figure measurements [46], these results demonstrate that the film is an untwinned single crystal.

$\mathrm{SnO}$ unit cell dimensions are determined by measuring interatomic distances in Fig. 3(a) and independently confirmed via high-resolution XRD reciprocal space maps (RSMs). Figure 3(c) is a typical RSM of $\mathrm{SnO} 114$ and $\mathrm{Al}_{2} \mathrm{O}_{3} 4 \overline{2} \overline{2} 6$ reflections. The film peak is centered at $k_{x}=$ $3.722 \mathrm{~nm}^{-1}$ and $k_{z}=8.264 \mathrm{~nm}^{-1}$, yielding a fully relaxed $\mathrm{SnO}$ unit cell with in-plane and out-of-plane lattice parameters of $a=\sqrt{2} / k_{x}=0.3800 \pm 0.0004 \mathrm{~nm}$ and $c=$ $4 / k_{z}=0.4840 \pm 0.0005 \mathrm{~nm}$. The centroid of the $\mathrm{Al}_{2} \mathrm{O}_{3} 4 \overline{2} 26$ reflection lies at $k_{x}=4.205 \mathrm{~nm}^{-1}$ and $k_{z}=8.619 \mathrm{~nm}^{-1}$, corresponding to effective lattice parameters ${ }^{2}$ of $a_{\mathrm{Al}_{2} \mathrm{O}_{3}}=$ $\sqrt{2} / k_{x}=0.3363 \mathrm{~nm}$ and $c_{\mathrm{Al}_{2} \mathrm{O}_{3}}=3 / k_{z}=0.3480 \mathrm{~nm}$. Based on the resulting film/substrate lattice parameter mismatch, $m=a_{\mathrm{Al}_{2} \mathrm{O}_{3}} / a-1=-12 \%$, the critical thickness [47] for strain relaxation is estimated to be less than one monolayer.

The relaxation of the $\mathrm{SnO}$ overlayer produces a semicoherent heteroepitaxial interface comprised of a periodic array of misfit dislocations. The dislocation cores are exposed by in-plane strain isocontours computed ${ }^{3}$ [48] from and overlaid on Fig. 3(a) (the raw data without the overlay are provided in Ref. [46]). Dislocation cores are found to be separated on average by $2.4 \mathrm{~nm}$, in excellent agreement with $a_{\mathrm{Al}_{2} \mathrm{O}_{3}} / m=$ $2.5 \mathrm{~nm}$, the expected dislocation line spacing for a fully relaxed $\mathrm{SnO}(001)$ film on $\mathrm{Al}_{2} \mathrm{O}_{3}(1 \overline{1} 02)$.

Despite the relaxed film structure, XRD $\theta-2 \theta$ thickness oscillations [Fig. 3(d)] and overlapping $\omega$-rocking curve film and substrate peaks [Fig. 3(e)] establish that the $\mathrm{SnO}$ layer exhibits a high degree of structural perfection. In-plane and out-of-plane mosaic coherence lengths [49], $\xi_{\|}=5 \mu \mathrm{m}$ and $\xi_{\perp} \simeq 40 \mathrm{~nm}$, are determined to be limited only by the intrinsic substrate mosaicity and finite film thickness, respectively. The high structural quality of the film is consistent with the orderly arrangement of atomic columns observed via latticeresolution STEM [Fig. 3(a)] and attributed to the formation of an intermediary interfacial structure.

Near the substrate region, high-resolution STEM images, including Fig. 3(f), show that the $\mathrm{SnO}$ film is divided into a commensurately strained monolayer and a fully relaxed overlayer. Separating the two sections is a $0.40 \pm$ 0.03 -nm-wide gap (75\% larger than interatomic distances

${ }^{2}$ Effective substrate lattice parameters are redefined along $\langle 202 \overline{1}\rangle$ and $\langle 1 \overline{1} 02\rangle$.

${ }^{3}$ Local strain fields

$$
\epsilon(\vec{r})=\frac{-1}{2 \pi} \sum_{\vec{g}} \vec{d}_{\vec{g}} \cdot \vec{\nabla}_{\vec{r}}\left\{\angle I_{\vec{g}}(\vec{r})-2 \pi \vec{g} \cdot \vec{r}\right\}
$$

are determined by applying the real-space gradient operator $\vec{\nabla}$ to the argument of the $\vec{g}$-filtered image $I_{g}(\vec{r})=\mathcal{F}_{\vec{g}}^{-1}\{\mathcal{F}[I(\vec{r})](\vec{k})\}(\vec{r})$ and taking the dot product of the result with $\vec{d}_{\vec{g}}$, the conjugate of $\vec{g}(\mathcal{F}$ is the Fourier transform operator). The phase ambiguity is removed by evaluating the gradient of the phase field $\psi(\vec{r})$ on the complex plane using $\partial \psi(\vec{r})=\operatorname{Im}\left\{e^{-i \psi(\vec{r})} \partial e^{i \psi(\vec{r})}\right\}$. 

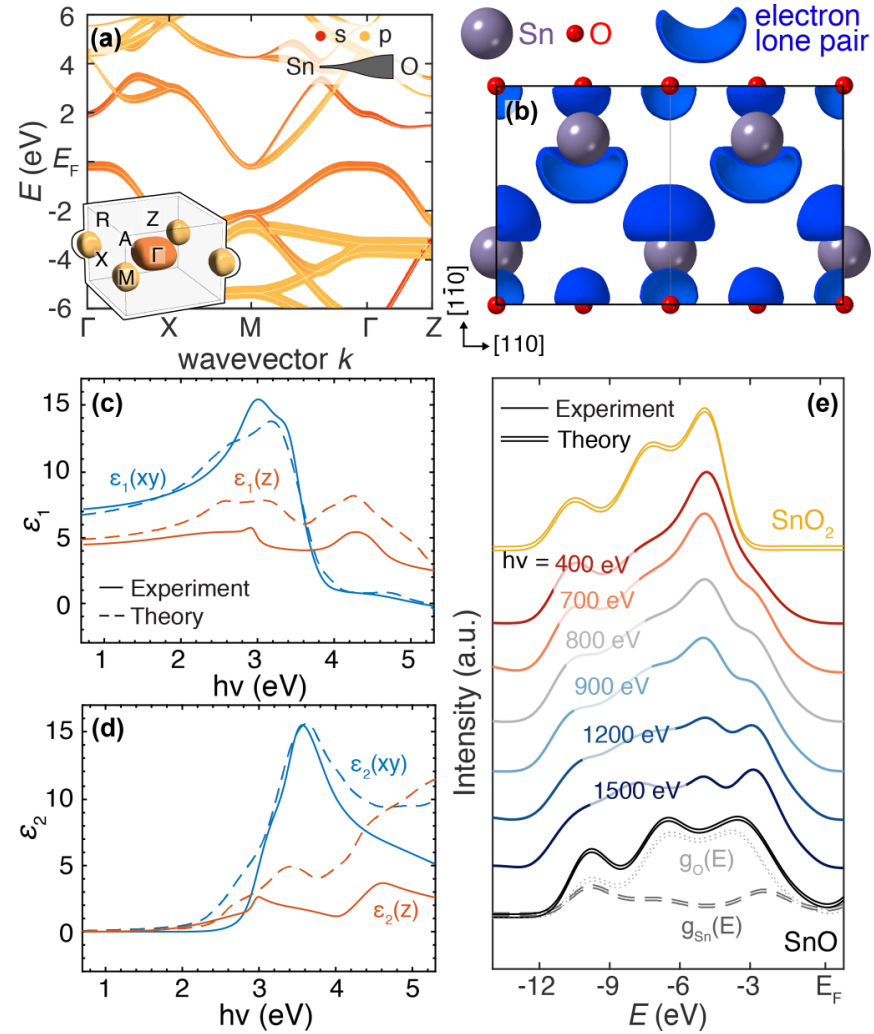

FIG. 4. Electronic properties of litharge $\mathrm{SnO}$, a model lone-pair system. (a) Theoretical $\mathrm{SnO}$ electronic band dispersions with states colorized and broadened according to orbital ( $s$ vs $p$ ) and atomic (tin vs oxygen) characters. The insert shows electron and hole pockets. (b) Charge-density maps of hole-pocket states reveal a lone-pairlike distribution. (c) and (d) $\mathrm{SnO}$ complex dielectric function $\varepsilon \equiv$ $\varepsilon_{1}+i \varepsilon_{2}$ resolved into ordinary $x y$ (blue) and extraordinary $z$ (red) components as determined from VASE (solid) and RPA calculations (dashed). (e) XPS scans as a function of photon energies between 400 and $1500 \mathrm{eV}$; the densities of states of $\mathrm{SnO}$ and $\mathrm{SnO}_{2}$ are also plotted for reference.

in $\mathrm{SnO}$ ) across which only weak van der Waals interactions are active [46]. These features are a hallmark of van der Waals epitaxy [50] whereby a weakly-bonded gap develops accommodating misfit dislocations and promoting films with high structural perfection despite a large lattice mismatch. Similar interfacial structures have been reported for $\mathrm{Bi}_{2} \mathrm{Te}_{3} / \mathrm{GaAs}(001)$ [51], $\mathrm{MoS}_{2} / \mathrm{GaN}(0001)$ [52], and $\mathrm{GaSe} / \mathrm{Si}(111)[53,54]$ heteroepitaxial systems, showing them to be common for the epitaxial integration of two-dimensional layered materials (e.g., SnO) on three-dimensional systems (e.g., $\mathrm{Al}_{2} \mathrm{O}_{3}$ ).

\section{Electronic and optical properties}

The electronic structure of $\mathrm{SnO}$ is investigated using a combination of transport measurements, variable-angle spectroscopic ellipsometry (VASE), and synchrotron x-ray photoelectron spectroscopy (XPS). Findings are interpreted within the context of band dispersions, charge-density distributions, and electronic densities of states computed from first principles density functional theory.
Figure 4(a) shows calculated $\mathrm{SnO}$ band dispersions, colorized and broadened according to orbital and atomic characters, along high-symmetry reciprocal-space directions. The valence-band maximum and conduction-band minimum occur along $\overline{M \Gamma}$ and at $M$, respectively, and give rise to the hole and electron pockets shown inscribed within the first Brillouin zone in Fig. 4(a). The hole pocket has strong contributions from $\mathrm{Sn}$ antibonding states which assume an asymmetric lonepair-like charge distribution [see Fig. 4(b)].

The lone-pair states profoundly influence the equilibrium unit-cell geometry. Rather than adopting the ideal $\mathrm{CsCl}$ structure for which the axial ratio $c / a=1$, the $\mathrm{SnO}$ cell is tetragonally elongated into the litharge structure $(c / a=1.27)$ as a result of the electronic pressure applied by the lone-pair states $[55,56]$.

The transport and optical properties of $\mathrm{SnO}$ are also affected by the lone-pair states. The room-temperature electrical resistivity of the $\mathrm{SnO} / \mathrm{Al}_{2} \mathrm{O}_{3}(1 \overline{1} 02)$ film is determined in the $\mathrm{SnO}(001)$ plane from four-point probe measurement [57] using pressed indium contacts to be $101 \Omega \mathrm{cm}$. Hall measurements carried out over an applied magnetic field range of $\mu_{o} H= \pm 6 \mathrm{~T}$ indicate hole conduction with a mobility of $2.4 \mathrm{~cm}^{2} \mathrm{~V}^{-1} \mathrm{~s}^{-1}$ and a carrier density of $2.5 \times 10^{16} \mathrm{~cm}^{-3}$ at room temperature. The measured carrier density value, which is the lowest reported to date [27], indicates trace levels of impurities and tin vacancies, a native mechanism known to engender holes [58], and suggests intrinsic phonon-limited transport. The hole mobility, which is smaller than values reported for polycrystalline films [27], is understood from curvature anisotropies in the lone-pair hole pocket [see Fig. 4(a)], which result in effective masses that are large in the $x y$ plane and small along $z$ the tetragonal axis.

$\mathrm{SnO}$ optical properties are probed via $\mathrm{VASE}^{4}$ [59]. The complex dielectric function $\varepsilon \equiv \varepsilon_{1}+i \varepsilon_{2}$ is plotted as a function of photon energy $h v$ in Figs. 4(c) and 4(d). Prominent poles, corresponding to optical excitations, are observed at 3.0 $(z), 3.6(x y)$, and $4.6 \mathrm{eV}\left(z^{\prime}\right)$; absorption is strongly suppressed below $2.7 \mathrm{eV}$, the direct optical gap, but remain finite down to $\sim 1 \mathrm{eV}$, the indirect optical gap. These features are reproduced by first principles calculations based on the random phase approximation (RPA) and indicate that the optical properties of $\mathrm{SnO}$ are well described by single-particle-like behavior. The combined experimental and theoretical results reveal that the high degree of optical transparency below the direct gap results from the small optical matrix element involving indirect excitations between lone-pair states [see Fig. 4(b)] and the conduction-band minimum [see Fig. 4(a)].

Figure 4(e) shows x-ray photoelectron spectroscopy valence-band scans collected as a function of photon energy $h v$ at beamline 29-ID of the Advanced Photon Source; computed densities of states corresponding to $\mathrm{SnO}$ and $\mathrm{SnO}_{2}$ are also shown. Spectra acquired at $h v=1500 \mathrm{eV}$ exhibit broad valence states spanning $12 \mathrm{eV}$ below the Fermi level.

\footnotetext{
${ }^{4}$ Ellipsometric angles are measured at $45^{\circ}, 65^{\circ}$, and $75^{\circ}$ incidences and modeled as a three-layer heterostructure comprised of a semiinfinite $\mathrm{Al}_{2} \mathrm{O}_{3}$ substrate, an anisotropic $\mathrm{SnO}$ layer with variable ordinary $x y$ and extraordinary $z$ dielectric responses, and a porous layer representing surface roughness.
} 
TABLE I. Summary of experimentally determined physical properties measured from a phase-pure, untwinned, relaxed, epitaxial litharge $\mathrm{SnO} / \mathrm{Al}_{2} \mathrm{O}_{3}(1 \overline{1} 02)$ layer grown via molecular-beam epitaxy at $380{ }^{\circ} \mathrm{C}$ in an $\mathrm{O}_{2}$ background partial pressure of $5 \times 10^{-7}$ Torr. Values obtained from first-principles calculations are shown in parentheses.

\begin{tabular}{|c|c|}
\hline $\mathrm{SnO} / \mathrm{Al}_{2} \mathrm{O}_{3}(1 \overline{1} 02)$ film properties & Value \\
\hline \multicolumn{2}{|l|}{ Lattice parameters } \\
\hline In-plane $a$ & $0.3800(0.3807) \mathrm{nm}$ \\
\hline Out-of-plane $c$ & $0.4840(0.4804) \mathrm{nm}$ \\
\hline Axial ratio $c / a$ & $1.27(1.26)-$ \\
\hline Film/substrate mismatch $m$ & $-12 \%$ \\
\hline \multicolumn{2}{|l|}{ Raman-active mode energies $\hbar \omega$} \\
\hline$E_{g}$ & $13.7(14.2) \mathrm{meV}$ \\
\hline$A_{1 g}$ & $25.8(25.8) \mathrm{meV}$ \\
\hline$B_{1 g}$ & $-(42.6) \mathrm{meV}$ \\
\hline$E_{g}^{\prime}$ & $-(56.3) \mathrm{meV}$ \\
\hline \multicolumn{2}{|l|}{ Dielectric function poles } \\
\hline$\epsilon(z)$ & $3.0(3.5) \mathrm{eV}$ \\
\hline$\epsilon(x y)$ & $3.6(3.7) \mathrm{eV}$ \\
\hline$\epsilon\left(z^{\prime}\right)$ & $4.6(5.0) \mathrm{eV}$ \\
\hline \multicolumn{2}{|l|}{ Band-gap energies $E_{\mathrm{g}}$} \\
\hline Indirect & $\sim 1(<0)$ eV \\
\hline Direct & $2.7(2.6) \mathrm{eV}$ \\
\hline \multicolumn{2}{|l|}{$p$-type transport properties } \\
\hline Resistivity $\rho$ & $101 \Omega \mathrm{cm}$ \\
\hline Hole concentration $p$ & $2.5 \times 10^{16} \mathrm{~cm}^{-3}$ \\
\hline Hole mobility $\mu_{p}$ & $2.4 \mathrm{~cm}^{2} \mathrm{~V}^{-1} \mathrm{~s}^{-1}$ \\
\hline \multicolumn{2}{|l|}{ Structural attributes } \\
\hline Mass density $\rho_{\mathrm{d}}$ & $6.2 \mathrm{~g} / \mathrm{cm}^{3}$ \\
\hline van der Waals gap $\delta$ & $0.3983 \mathrm{~nm}$ \\
\hline Surface roughness $\rho_{\text {rms }}$ & $1.0 \mathrm{~nm}$ \\
\hline Screw dislocation density $\rho_{\mathrm{s}}$ & $5 \times 10^{9} \mathrm{~cm}^{-2}$ \\
\hline \multicolumn{2}{|l|}{ Mosaic coherence lengths } \\
\hline In-plane $\xi_{\|}$ & $5 \mu \mathrm{m}$ \\
\hline Out-of-plane $\xi_{\perp}$ & $\sim 40 \mathrm{~nm}$ \\
\hline
\end{tabular}

In addition, two peaks of approximately equal intensity are visible at -5.0 and $-2.5 \mathrm{eV}$. As the photon energy is decreased, spectral weights shifts from the peak at $-2.5 \mathrm{eV}$ to the one at $-5.0 \mathrm{eV}$. This evolution in spectral weight cannot be explained by an energy-dependent matrix element [60]. The differences are instead attributed to a sensitivity that changes with depth. This results from the combination of a varying photoelectron inelastic mean-free path $\ell$ (for $h v=$ $400 \mathrm{eV}, \ell \leqslant 1 \mathrm{~nm}$; at $h v=1500 \mathrm{eV}, \ell \sim 4 \mathrm{~nm}$ ) [61] and the presence of a thin $(\lesssim 4 \mathrm{~nm})$ native $\mathrm{SnO}_{2}$ layer on the air-exposed surface of the $\mathrm{SnO}$ film.

\section{CONCLUSIONS}

Despite the wide range of desirable properties associated with $\mathrm{Sn}^{2+}$, tin generally prefers to adopt a $4+$ oxidation state, making the stabilization of the former valence challenging. We successfully demonstrate the growth of epitaxial $\mathrm{SnO}$ layers with the litharge structure on $\mathrm{Al}_{2} \mathrm{O}_{3}(1 \overline{1} 02)$ using molecular-beam epitaxy. In addition to quantifying the structural perfection and identifying the growth modality of the layers, we report the physical properties of our epitaxial $\mathrm{SnO}$ films. Our main results are summarized in Table I.

\section{ACKNOWLEDGMENTS}

The authors thank Jessica McChesney, beamline scientist at the Advanced Photon Source, for her assistance. A.B.M., Z.W., M.B., L.E.N., and D.G. Schlom acknowledge support from ASCENT, one of six centers in JUMP, a Semiconductor Research Corporation (SRC) program sponsored by DARPA. N.J.S. acknowledges support from the National Science Foundation (NSF) Graduate Research Fellowship Program under Grant No. DGE-1650441. This work made use of the Cornell Center for Materials Research (CCMR) Shared Facilities, which are supported through the NSF MRSEC Program (No. DMR-1719875). Substrate preparation was performed in part at the Cornell NanoScale Facility, a member of the National Nanotechnology Coordinated Infrastructure (NNCI), which is supported by the NSF (Grant No. ECCS-1542081). This work was carried out in part in the Frederick Seitz Materials Research Laboratory Central Facilities, University of Illinois. M.J.W. and L.F.J.P. acknowledge support from the Air Force Office of Scientific Research under award number FA955018-1-0024. This research used resources of the Advanced Photon Source, a U.S. Department of Energy (DOE) Office of Science User Facility operated by the DOE Office of Science by Argonne National Laboratory under Contract No. DEAC02-06CH11357; additional support by NSF under Grant no. DMR-0703406. H.P. acknowledges support from the NSF [Platform for the Accelerated Realization, Analysis, and Discovery of Interface Materials (PARADIM)] under Cooperative Agreement No. DMR-1539918. D.G. Sangiovanni gratefully acknowledges financial support from the Olle Engkvist Foundation and access to supercomputer resources from the Swedish National Infrastructure for Computing (SNIC).
[1] J. F. Wager, D. A. Keszler, and R. E. Presley, Transparent Electronics (Springer Science \& Business Media, Berlin, 2007).

[2] H. J. Kim, U. Kim, H. M. Kim, T. H. Kim, H. S. Mun, B.-G. Jeon, K. T. Hong, W.-J. Lee, C. Ju, K. H. Kim, and K. Char, Appl. Phys. Express 5, 061102 (2012).

[3] Z. Chen, W. Li, R. Li, Y. Zhang, G. Xu, and H. Cheng, Langmuir 29, 13836 (2013).

[4] H. Paik, Z. Chen, E. Lochocki, A. Seidner H, A. Verma, N. Tanen, J. Park, M. Uchida, S. Shang, B.-C. Zhou, M. Brützam,
R. Uecker, Z.-K. Liu, D. Jena, K. M. Shen, D. A. Muller, and D. G. Schlom, APL Mater. 5, 116107 (2017).

[5] G. Hautier, A. Miglio, G. Ceder, G.-M. Rignanese, and X. Gonze, Nat. Commun. 4, 2292 (2013).

[6] G. Hautier, A. Miglio, D. Waroquiers, G.-M. Rignanese, and X. Gonze, Chem. Mater. 26, 5447 (2014).

[7] V.-A. Ha, F. Ricci, G.-M. Rignanese, and G. Hautier, J. Mater. Chem. C 5, 5772 (2017).

[8] M. Seth, K. Faegri, and P. Schwerdtfeger, Angew. Chem., Int. Ed. 37, 2493 (1998). 
[9] A. Prakash, P. Xu, A. Faghaninia, S. Shukla, J. W. Ager, C. S. Lo, and B. Jalan, Nat. Commun. 8, 15167 (2017).

[10] S. S. Shin, E. J. Yeom, W. S. Yang, S. Hur, M. G. Kim, J. Im, J. Seo, J. H. Noh, and S. I. Seok, Science 356, 167 (2017).

[11] P.-J. Chen and H.-T. Jeng, Sci. Rep. 5, 032113 (2015).

[12] M. K. Forthaus, K. Sengupta, O. Heyer, N. E. Christensen, A. Svane, K. Syassen, D. I. Khomskii, T. Lorenz, and M. M. AbdElmeguid, Phys. Rev. Lett. 105, 157001 (2010).

[13] Q.-Y. Wang, Z. Li, W.-H. Zhang, Z.-C. Zhang, J.-S. Zhang, W. Li, H. Ding, Y.-B. Ou, P. Deng, K. Chang, J. Wen, C.-L. Song, K. He, J.-F. Jia, S.-H. Ji, Y.-Y. Wang, L.-L. Wang, X. Chen, X.-C. Ma, and Q.-K. Xue, Chin. Phys. Lett. 29, 037402 (2012).

[14] P. O. Sprau, A. Kostin, A. Kreisel, A. E. Böhmer, V. Taufour, P. C. Canfield, S. Mukherjee, P. J. Hirschfeld, B. M. Andersen, and J. C. S. Davis, Science 357, 75 (2017).

[15] Y. Ogo, H. Hiramatsu, K. Nomura, H. Yanagi, T. Kamiya, M. Hirano, and H. Hosono, Appl. Phys. Lett. 93, 032113 (2008).

[16] J. A. Caraveo-Frescas, P. K. Nayak, H. A. Al-Jawhari, D. B. Granato, U. Schwingenschlögl, and H. N. Alshareef, ACS Nano 7, 5160 (2013).

[17] Z. Wang, X. He, X.-X. Zhang, and H. N. Alshareef, Adv. Mater. 28, 9133 (2016).

[18] K. J. Saji, K. Tian, M. Snure, and A. Tiwari, Adv. Electron. Mater. 2, 1500453 (2016).

[19] Y. Idota, T. Kubota, A. Matsufuji, Y. Maekawa, and T. Miyasaka, Science 276, 1395 (1997).

[20] F. Zhang, J. Zhu, D. Zhang, U. Schwingenschlögl, and H. N. Alshareef, Nano Lett. 17, 1302 (2017).

[21] J. Geurts, S. Rau, W. Richter, and F. J. Schmitte, Thin Solid Films 121, 217 (1984).

[22] V. Kraševec, Z. Škraba, M. Hudomalj, and S. Sulčič, Thin Solid Films 129, L61 (1985).

[23] X. Q. Pan and L. Fu, J. Appl. Phys. 89, 6048 (2001).

[24] W. Guo, L. Fu, Y. Zhang, K. Zhang, L. Y. Liang, Z. M. Liu, H. T. Cao, and X. Q. Pan, Appl. Phys. Lett. 96, 042113 (2010).

[25] X. Q. Pan and L. Fu, J. Electroceram. 7, 35 (2001).

[26] H. Hayashi, S. Katayama, R. Huang, K. Kurushima, and I. Tanaka, Phys. Status Solidi RRL 9, 192 (2015).

[27] Z. Wang, P. K. Nayak, J. A. Caraveo-Frescas, and H. N. Alshareef, Adv. Mater. 28, 3831 (2016).

[28] T. Wang, K. C. Pitike, Y. Yuan, S. M. Nakhmanson, V. Gopalan, and B. Jalan, APL Mater. 4, 126111 (2016).

[29] T. Fix, S. L. Sahonta, V. Garcia, J. L. MacManus-Driscoll, and M. G. Blamire, Cryst. Growth Des. 11, 1422 (2011).

[30] B. Predel, O-Sn (Oxygen-Tin), Landolt-Börnstein - Group IV Physical Chemistry Vol. I (Springer-Verlag, Berlin/Heidelberg, 1998).

[31] R. H. Lamoreaux and D. L. Hildenbrand, J. Phys. Chem. Ref. Data 16, 419 (1987).

[32] M. Y. Tsai, M. E. White, and J. S. Speck, J. Appl. Phys. 106, 024911 (2009).

[33] P. Vogt and O. Bierwagen, Appl. Phys. Lett. 106, 081910 (2015).
[34] T. C. Damen, S. P. S. Porto, and B. Tell, Phys. Rev. 142, 570 (1966).

[35] D. Porezag and M. R. Pederson, Phys. Rev. B 54, 7830 (1996).

[36] D. R. Hamann, X. Wu, K. M. Rabe, and D. Vanderbilt, Phys. Rev. B 71, 035117 (2005).

[37] J. B. Nelson and D. P. Riley, Proc. Phys. Soc. 57, 160 (1945).

[38] H. M. Rietveld, J. Appl. Cryst. 2, 65 (1969).

[39] F. Izumi, J. Solid State Chem. 38, 381 (1981).

[40] H. Kiessig, Ann. Phys. 402, 769 (1931).

[41] Y. Yoneda, Phys. Rev. 131, 2010 (1963).

[42] L. G. Parratt, Phys. Rev. 95, 359 (1954).

[43] G. Ehrlich and F. G. Hudda, J. Chem. Phys. 44, 1039 (1966).

[44] R. L. Schwoebel and E. J. Shipsey, J. Appl. Phys. 37, 3682 (1966).

[45] S. K. Sinha, E. B. Sirota, S. Garoff, and H. B. Stanley, Phys. Rev. B 38, 2297 (1988).

[46] See Supplemental Material at http://link.aps.org/supplemental/ 10.1103/PhysRevMaterials.3.105202 for additional film characterization results, including XRD pole figures, as-captured STEM micrographs, in situ RHEED patterns, and temperaturedependent transport data.

[47] J. W. Matthews and A. E. Blakeslee, J. Cryst. Growth 27, 118 (1974).

[48] M. J. Hÿtch, E. Snoeck, and R. Kilaas, Ultramicroscopy 74, 131 (1998).

[49] A. B. Mei, B. M. Howe, C. Zhang, M. Sardela, J. N. Eckstein, L. Hultman, A. Rockett, I. Petrov, and J. E. Greene, J. Vac. Sci. Technol. A 31, 061516 (2013).

[50] A. Koma, Thin Solid Films 216, 72 (1992).

[51] J. Houston Dycus, R. M. White, J. M. Pierce, R. Venkatasubramanian, and J. M. LeBeau, Appl. Phys. Lett. 102, 081601 (2013).

[52] T. P. O'Regan, D. Ruzmetov, M. R. Neupane, R. A. Burke, A. A. Herzing, K. Zhang, A. G. Birdwell, D. E. Taylor, E. F. C. Byrd, S. D. Walck, A. V. Davydov, J. A. Robinson, and T. G. Ivanov, Appl. Phys. Lett. 111, 051602 (2017).

[53] L. E. Rumaner, J. Vac. Sci. Technol. B 16, 977 (1998).

[54] N. Jedrecy, R. Pinchaux, and M. Eddrief, Phys. Rev. B 56, 9583 (1997).

[55] G. W. Watson, J. Chem. Phys. 114, 758 (2001).

[56] A. Walsh, D. J. Payne, R. G. Egdell, and G. W. Watson, Chem. Soc. Rev. 40, 4455 (2011).

[57] L. J. van der Pauw, Philips Tech. Rev. 20, 220 (1958).

[58] A. Togo, F. Oba, I. Tanaka, and K. Tatsumi, Phys. Rev. B 74, 195128 (2006).

[59] D. A. G. Bruggeman, Ann. Phys. 416, 636 (1935).

[60] N. F. Quackenbush, J. P. Allen, D. O. Scanlon, S. Sallis, J. A. Hewlett, A. S. Nandur, B. Chen, K. E. Smith, C. Weiland, D. A. Fischer, J. C. Woicik, B. E. White, G. W. Watson, and L. F. J. Piper, Chem. Mater. 25, 3114 (2013).

[61] S. Tanuma, C. J. Powell, and D. R. Penn, Surf. Interface Anal. 37, 1 (2005). 